\title{
Die täglichen Gebetsstunden im Judentum und Urchristentum.
}

\author{
Von O. Holtzmann in GieBen.
}

1. Harnack hat in seiner großen Ausgabe der Didache (1884) dem

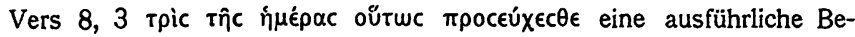
sprechung gervidmet (S. 27 f.). Er betont, daß an der genannten Stelle zum erstenmal in der kirchlichen Literatur geboten sei, dreimal des Tages $\mathrm{zu}$ beten, und zwar dreimal dảs Vaterunser $\mathrm{zu}$ beten. „Aus Tertullian de orat. 25 und de jejun. 10 entnahm man bislang das älteste Zeugnis für die dritte, sechste und neunte Stunde als Gebetsstunden." Aber Tertullian fügt hinzu, „daß daneben das Morgen- und Abendgebet zu halten sei." Harnack schwankt, ob der Verfasser der Didache auch schon die dritte, sechste und neunte Stunde gemeint hat oder den Morgen, Nachmittag und Abend als Gebetszeiten betrachtet, im Anschluß an die - nach Schürer festgestellte - jüdische Sitte, das Schmone Esre morgens, nachmittags und abends $z \mathrm{u}$ beten. „Für die Anlehnung an diese Sitte spricht, daß nach Tertullian unzweifelhaft die Sitte, um die dritte, sechste und neunte Stunde $z \mathrm{u}$ beten, jüngeren Ursprungs in der Kirche ist als die Sitte, die, legitimæ orationes' am Morgen und Abend zu halten. - Ferner spricht für die Anlehnung an die jüdische Sitte, daß unser Verfasser ein bestimmtes Gebet, eben das Vaterunser, vorschreibt." Harnack meint, ein Vergleich von Tertullian de orat. 25 und 10 lasse es als nicht unwahrscheinlich erkennen, ${ }_{n}$ daß eben am Abend und Morgen das Vaterunser gebetet werden mußte. Damit wird es noch einmal unsicher, ob die Didache die dritte, sechste und neunte Stunde gemeint hat (so allerdings die Apostolischen Konstitutionen und spätere Bestimmungen)". Zum Schluß hebt es Harnack als sehr beachtenswert hervor, daß Origenes de orat. 12 auch von drei Gebetszeiten am Tage spricht, aber den Morgen, Mlittag und Abend nennt, also nicht die dritte, sechste und neunte Stunde. Das scheine alexandrinische Praxis gewesen zu sein, wogegen eine von Bryennios aus 
dem 7. Buch der Stromateis des Clemens angeführte Stelle nichts beweise, da Clemens das Beten zur dritten, sechsten und neunten Stunde nur als eine Ordnung unter mehreren anführt.

Diese Sätze Harnacks bieten eine Fülle von Anregung zu wissenschaftlicher Arbeit; eine Entscheidung wollen sie selbst nicht geben. Offenbar im Anschluß an Harnack sagt P. Drews in seinen Anmerkungen zur Didache in Henneckes Handbuch zu den Neutestanientlichen Apolkryphen S. $268 \mathrm{zu} \mathrm{8,3}$ "Wie in der Fastensitte, so blieben auch in der Gebetssitte Christen dem jüdischen Gebrauche treu. Höchst wahrscheinlich ist bei dem dreimaligen Beten des Vaterunsers am Tage nicht an die spätern christlichen Gebetsstunden (dritte, sechste und neunte Stunde), sondern an die jüdischen Gebetsstunden morgens, mittags und abends $z \mathrm{u}$ denken, bei weichen das Schmone Esre von den Juden gebetet wurde. An die Stelle dieses Gebetes soll bei den Christen das Vaterunser treten. Es ist durchaus möglich, daß auch Judenchristen noch das Schmone Esre an den Gebetsstunden $\mathrm{zu}$ beten pflegten. Das soll ihnen verleidet werden, indem die so betenden Juden als ,Heuchler" bezeichnet werden."

Drews setzt also für Harnacks vorsichtigen Ausdruck „nicht unwahrscheinlich" ein mutiges "höchst wahrscheinlich“; er bestimmt auch die jüdischen Gebetsstunden anders, als Harnack nach Schürer (Gesch. des jüd. Volkes im Zeitalter Jesu Christi, jetzt 4. Aufl., II, 350, 40) tut. Harnack bzw. Schürer bezeichnen als jüdische Gebetszeiten Morgen, Nachmittag und Abend; Drews dagegen Morgen, Mittag und Abend. Bei einem pünktlichen Arbeiter, wie Drews es ist, darf man kein bloßes Versehen annehmen; tatsächlich hat er, wie wir zeigen werden, zu seiner Angabe über die jüdischen Gebetszeiten guten Grund. Nur wäre es wünschenswert gewesen, daß er seine Auffassung durch kurzen Quellennachw'eis begründet hätte, da sie sonst gegen die Autorität Schürers auf diesem Gebiete nicht aufkommt. Dagegen hat Drews schiverlich recht, wenn er annimmt, daß Judenchristen am Schmone Esre festhielten. Das Achtzehngebet stammt aus der Zeit nach der Zerstörung Jerusalems. Das zeigt seine ältere palästinische Gestalt, wie sie neuerdings in Ägypten gefunden wurde (herausgegeben von Dalman, Worte Jesu, 1898, S. 299. 300). Auch in der Mischna (Berachoth $4,3.4$ a) streiten die Autoritäten um $100 \mathrm{n}$. Chr. noch darüber, ob das Achtzehngebet regelmäßig zu beten sei.

2. Tertullian empfiehlt de orat. 25 als Gebetszeiten die dritte, sechste und neunte Stunde ohne Hinweis auf eine entsprechende jüdische Sitte, nur mit Bezugnahme auf die Ausgießung des hl. Geistes 
um die dritte Stunde (Act 2,15), auf die Offenbarung an Petrus bei seinem Gebet um die sechste Stunde (Act 10,9) und auf die Heilung des Lahmen beim Besuch des Tempels um die neunte Stunde (Act 3,1). Tertullian betont dann den Segen einer festen Gewöhnung und verweist dabei auf das Beispiel Daniels, der nach jüdischer Ordnung dreimal gebetet hat (Dan 6, 10); nach seinem Ausdruck „observatum utique ex Israelis disciplina" scheint Tertullian diese jüdische Ordnung nicht näher zu kennen. Weniger als dreimal aber, meint er, dürfen die nicht beten, welche drei Personen (Vater, Sohn und Geist) verpflichtet sind „abgesehen von den gesetzlichen Gebeten, die man ohne jedes Mahnwort bei Eintritt von Tag und Nacht zu sprechen schuldig ist". Außerdem dürfen Gläubige nicht ohne Gebet eine Speise oder ein Bad nehmen.

Auf dieselben Bibelstellen wie de orat. 25 verweist auch Tertullian, de jejun. 10, um die Notwendigkeit eines Gebetes zu bestimmten Tagesstunden, und zwar der dritten, sechsten und neunten $z u$ erweisen. Hier weiß er aber Genaueres über die jüdische Sitte. Er sagt, daß Petrus nach Act 3,1 die neunte Stunde als letzte Gebetsstunde einem alten Gebrauche gemäß gehalten habe: hinc itaque et Petrum dicam ex vetere potius usu observasse tertio orantem supremae orationis munere. Also das Gebet um die neunte Stunde ist das dritte (tertium munus) und letzte Tagesgebet (suprema oratio). Diese Anschauung der jüdischen Sitte paßt weder zu der Anschauung von Schürer noch zu der von Drews; Schürer und Drews meinen, $\mathrm{da} B$ das dritte Gebet erst abends gesprochen wurde.

Ubrigens empfiehlt Tertullian das Gebet um die dritte, sechste und neunte Stunde an beiden angeführten Stellen auch aus einem Grund in der Sitte der römischen Welt. Er nennt diese Stunden de orat. 25 istae communes, quae diei interspatia signant, und de jejun. 10 bezeichnet er sie als insigniores in rebus humanis, quae diem distribuunt, quae negotia distinguunt, quae publice resonant. Das bezieht sich darauf, daß gerade diese drei Tagesstunden schon zur Zeit Varros öffentlich ausgerufen wurden (Varro, de lingua Latina VI, 89).

Nun sagt doch Tertullian, daß die drei Tagesgebete zu sprechen seien nabgesehen von den gesetzlich gebotenen bei Eintritt des Tages und der Nacht" (de orat. 25: exceptis utique legitimis orationibus, quae sine ulla admonitione debentur ingressu lucis et noctis). Harnack ging dem Ausdruck legitima oratio nach und fand ihn außer de orat. 25 auch de orat. 10, wo er zweifellos das Vaterunser bezeichnet. Tertullian sagt hier, daß der Christ je nach seiner besondern Lage bestimmte 
Einzelwünsche vor Gott bringen dürfe præmissa legitima et ordinaria oratione quasi fundamento. Also das Vaterunser muß jedem besondern Gebete vorausgehen.

Harnack hat also guten Grund, die Vermutung zu äußern, daß an beiden Stellen (de orat. 10 und 25) mit legitima oratio dasselbe gemeint sei und daß also das Vaterunser bei Eintritt von Tag und Nacht gesprochen wurde. Und doch ist das wohl ein Irrtum. Denn de orat. 10 ist das Vaterunser als das gesetzmäßige Gebet bezeichnet im Unterschied von Gelegenheitsgebeten, die aus der augenblicklichen Lage des Christen geboren sind; da bezieht sich also legitimus auf Inhalt und Form des Gebetes; das Vaterunser ist das immer gleiche Gebet des Herrn. Aber de orat. 25 sind die Gebete bei Eintritt von Tag und Nacht um dieses ihres Zeitpunktes willen als legitimae bezeichnet: um diese Zeit muB nach christlicher Ordnung gebetet werden, während es sich bei den drei Gebetsstunden deutlich nur um ein Herkommen handelt, das Tertullian empfiehlt, ohne doch ein Gesetz daraus zu machen. Nur eins verlangt er: es soll mindestens dreimal im Tage gebetet werden, und das Vaterunser muß der Grundstock dieser Gebete sein.

Tertullian will ja doch de orat. 25 und de jejun. 10 die Zeiten des täglichen Gebetes bestimmen und gibt deshalb beidemal für die von ihm gutgeheißenen Gebetsstunden eine ausführliche Begründung. Was in diesen drei Zeiten gesprochen wird, ist schlechthin „das Gebet“. Nur beiläufig und nachträglich besinnt er sich de orat. 25 auch auf die gesetzlichen Gebete, die bei Eintritt von Tag und Nacht pflichtmäßig seien. Also sind diese gesetzlichen Morgen- und Abendgebete gewiß nicht das Vaterunser. Das hätte Tertullian bei der Bedeutung, die er gerade auch de orat. 10 dem Vaterunser beimißt, nimmermehr nur so nachträglich erwähnt. Zudem wissen wir jetzt aus Did. 8, 3, daß das Vaterunser in der alten Kirche dreimal täglich gesprochen wurde. So werden wir nicht umhin können, anzunehmen, daß das Vaterunser nach Tertullian das in den drei von ihm empfohlenen Gebetsstunden gebräuchliche Hauptgebet war, daß aber bei Eintritt von Tag und Nacht andere Formeln ordnungsmäßig aufgesagt wurden.

3. Harnack hat zweifellos recht, wenn er für die christliche Sitte besonderer Gebetszeiten das Vorbild im Judentum sucht. Tatsächlich kehrt im gleichzeitigen Judentum alles wieder: ein Bekenntnis bei Anbruch des Tages und der Nacht gilt als gesetzlich vorgeschrieben, Davon ist zu unterscheiden das Gebet, das nach feststehender Sitte dreimal im Tage gesprochen werden soll.

Josephus führt antt. 4, 212 als Gebot Mosis an: „Zweimal jeden 
Tag, bci scinem Beginn und wenn es Zeit ist schlafen $z \mathrm{u}$ gehen, soll man Gott die Wohltaten bezeugen, die er denen gewährte, die er aus dem Lande der Ägypter befreite." Damit umschreibt Josephus in allerdings schr gekürzter Form das jüdjsche Bekenntnis, wie es täglich zweimal gesprochen wird und sich aus den Stellen Dt 6, 4-9;11, 13 bis 21 und Num 15, 34-41 zusammensetzt, das sogenannte Schma.

Dieses Bekenntnis betont nämlich in seinem Eingang die Einheit Gottes und die Pflicht der Gottesliebe: beides soll man sich zu Herzen nehmen und immer und überall einschärfen; man soll es auf Armund Stirnbänder und an die Türpfosten schreiben (Dt 6, 4-9). - Der Lohn des Gehorsams ist Fruchtbarkeit und fester Besitz des gelobten Landes; die Strafe des Ungehorsams Unfruchtbarkeit und Verlust dieses Bodens (Dt 11,13-21). - Auch die Quasten am Kleid sollen an die Gebote des Gottes erinnern, der Israel aus dem Lande Ägypten geführt hat (Num 15, 37-41).

Die Pflicht, dieses Bekenntnis zweimal täglich, morgens und abends, aufzusagen, entnimmt man seinen eigenen Worten. Dt 6, 7 heißt es: "sprich von (diesen Worten), wenn du weilst zu Hause, wenn du gehst auf der Straße, beim Liegen und Aufstehn." Ebenso Dt 11, 19: "lehrt sie eure Söhne, von ihnen zu reden zu Hause und auf der Straße, beim Liegen und Aufstehn." Es ist klar, daß sich daraus die Pflicht eines ausgerechnet zweimaligen Aufsagens irgendwelcher Formel nicht unmittelbar ergibt. Verlangt ist nur ein ununterbrochenes Gedenken an den Glauben und seine Forderung und ein Reden davon, sooft sich Gelegenheit dazu bietet. Mindestens verlangt keiner dieser Gesetzesabschnitte, in seinem ganzen Umfang immer wieder aufgesagt $z \mathrm{u}$ werden, und ebensowenig ist das sonstivo im jüdischen Gesetzbuch gefordert. Insbesondre der dritte Abschnitt von den Quasten am Kleid (Num 15, 34-41) verlangt nirgends das Aufsagen irgendwelcher Worte. Der Anschluß dieser Verse an. das Bekenntnis empfahl sich nur, weil so am leichtesten die Forderung erfüllbar schien, die (ursprünglich) violetten Quasten an den Enden des Gewandes anzuschauen und dabei der Gebote zu gedenken (Num 15,39).

Dieses jüdische Bekenntnis ist also im Gesetz nicht einfach gegeben; die Sitte, es zweimal täglich aufzusagen, ist keine Forderung des Gesetzes, wenn das auch gleich so behauptet wird. Die Formel ist irgendeinmal durch bewußte Arbeit aus den drei im Gesetzbuch nicht zusammenstehenden Abschnitten zusammengestellt worden. Ebenso ist irgendeinmal in bewußter Arbeit das zweimalige Aufsagen dieses Bekenntnisses im ganzen Gebiete des Judentums durchgesetzt worden. 
Keine Überlieferung meldet die Namen der Männer, die hier mitgewirkt haben. Josephus weiß es nicht anders, als daß man morgens und abends dieses Bekenntnis spricht; die jüdische Gemeinde muß an diesem Gebote Moses festhalten, auch nachdem ihr das Land genommen ist, dessen fester Besitz ihr nach dem Bekenntnis, unter der Bedingung der Gesetzestreue, von Gott zugesagt war. Jetzt war das Festhalten am Bekenntnis eine harte Aufgabe. Das empfindet Josephus, wenn er den Inhalt des Bekenntnisses in die Formel faßt: zweimal täglich soll man Gott die Wohltaten bezeugen, die er denen gewährt hat, die er aus dem Land Ägypten befreite (antt. 4,212).

Für Josephus stammt das Bekenntnis und die Pflicht es aufzusagen von Mose; Philo von Alexandrien kennt das Bekenntnis noch gar nicht. Philo hätte häufig Anlaß gehabt, die Stelle Dt 6, +.5 anzuführen, wenn sie ihm irgend geläufig gewesen wäre; denn er betont stark den Gedanken der Einheit Gottes (de mundi opif. 61; leg. alleg. 1, 14;

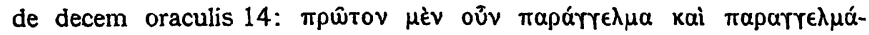

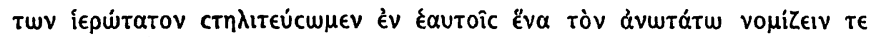

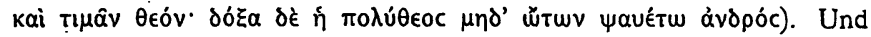
wie ihm Gotteserkenntnis und Ähnlichkeit mit Gott das höchste Ziel ist, so müßte ihm die Pflicht, mit ganzem Herzen, ganzer Seele und aller Macht Gott $z u$ lieben, ein äußerst willkommener Ausdruck seiner eigenen Gedanken gewesen sein; vgl. de decem orac. 15: єủxìv ảpictnv

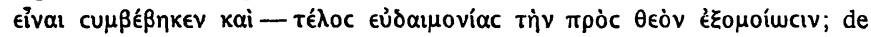

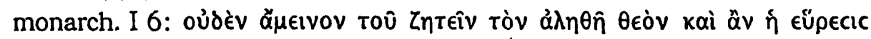

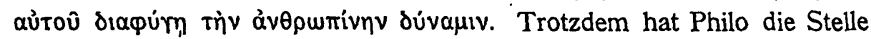
Dt 6, 4.5 in den uns überlieferten zahlreichen Werken nirgends angeführt.

Dagegen bespricht Philo die Stelle Dt 6, 6-9 in dem ersten Ka-

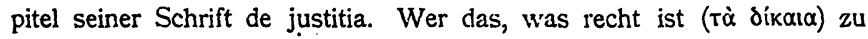
Herzen nimmt, an die Hand befestigt und sich vor Augen schweben läßt (cєเó $\mu \in v \alpha \pi \rho \dot{\delta} \delta \varphi \theta \alpha \lambda \mu \hat{\omega} v$ ), der ist ein vollkommener Mann und wird als solcher der Lehrer der andern. ${ }_{n}$ Er lehre also, was recht ist, Verwandte und Freunde und die Jugend zu Hause und unterwegs, wenn sie zu Bette gehen und aufstehen, damit sie in allen Lagen und Bewegungen, an allen eigenen und öffentlichen Plätzen nicht nur wachend, sondern auch schlafend sich an der Vorstellung dessen, was recht ist, erfreuen."

Hier ist also durchaus nicht die Rede vom regelmäßigen Aufsagen irgendwelcher Formel, sondern von der Belehrung der Angehörigen. Sie sollen immer gemahnt werden an das, was recht ist, auch wenn sie gerade zu Bette gehen oder aufstehen. Daß der Fromme für sich 
sein Bekenntnis $\mathrm{zu}$ sprechen hat, liegt Philo ganz ferne. Er kann also diese Sitte noch nicht geübt haben. Wäre er in der Gewohnheit, das Bekenntnis morgens und abends zu sprechen, aufgewachsen, so hälte er diese Deutung nicht vorbringen können. Dazu kommt also noch, daß er dic Worte von Gottes Einheit und der Pflicht der Gottesliebe (Dt 6, 4.5), so sehr sie in seinen Anschauungskreis passen, niemals erwähnt. Da Philo sicher den Tod des Caligula (41 n. Chr.) überlebt hat, wird man sagen dürfen: das Aufsagen des jüdischen Bekenntnisses war um $40 \mathrm{n}$. Chr. den Juden Alexandrias noch unbekannt.

Daran ändert auch nichts die Stelle des Aristeasbriefs, die auf Dt 6,6-9 Bezug nimmt (Wendland 157-160). Hier wird darauf hingewiesen, wie das Gesetz immer und überall Erinnerung an Gott fordert: beim Essen durch das Tischgebet, an den Kleidern durch die Quasten, an Toren und Türen durch Sprüche, an den Händen durch die Gebetsriemen. Dann heißt es $(\$ 160)$ : „Er befiehlt aber auch, beim Zubettegehen, Aufstehen und Gehen die Veranstaltungen Gottes zu verarbeiten ( $\mu \in \lambda \in \tau \hat{\alpha} v)$, nicht nur mit Worten, sondern mit Verständnis anschauend die eigne Bewegung und Vorstellung, wenn man zum Schlafe kommt, und das Erwachen, wie göttlich und unmerklich dieser Wechsel ist." Also Aristeas behauptet, das Gesetz fordere eine Beobachtung der physiologischen Vorgänge insbesondere beim Einschlafen und Erwachen; diese Forderung soll nicht bloß beim Zubettegehen und Aufstehen, sondern ebenso beim Gehen, d. h. wenn man irgendeinen Weg macht, erfüllt werden. Also die feste Sitte der spätern Zeit kennt der Aristeasbrief nicht. Von ihr aus wäre er zu einer solchen Verflüchtigung und Verschiebung der Gesetzesworte nicht gekommen. Läßt er doch auch die Denkzeichen an Kleidern, Türen und Gebetsriemen von Umdeutung frei. Die Gewohnheit, das Bekenntnis morgens und abends aufzusagen, ist ihm noch fremd.

Damit sind die Zeitgrenzen.enge zusammengerückt, innerhalb deren das Aufsagen des Bekenntnisses aufgekommen sein muß. Josephus, der das Bekenntnis als mosaisches Erbgut betrachtet, ist im ersten Jahr des Kaisers Gajus (Caligula) geboren (37 n. Chr. - vita 5 antt. 20, 267). Philo ist als alter Mann im Auftrag der Judenschaft Alexandrias zu diesem Kaiser gereist (leg. ad Gai.1.28); seine Erinnerungen daran hat er sicher erst nach des Gajus Tod (24. Januar $41 \mathrm{n}$. Chr.) veröffentlicht: und er kennt die Sitte des täglichen Bekenntnisses noch nicht. So müßte sich denn diese Sitte etwa $z$ wischen dem dritten und fünften Jahrzehnt des ersten christlichen Jahrhunderts zunächst in Palästina eingebürgert haben; von da aus verbreitet sie sich allmählich über die Diaspora. 
4. Diese Jahrzehnte sind aber auch die Zeit der Predigt Jesu und des ersten Aufkommens des Christentums. Von hier aus fällt ein besonderes Licht auf die Erzählung der synoptischen Evangelien, daß Jesus das Gebot Dt 6,4.5 für das erste im Gesetz erklärt habe: mit diesen Worten beginnt ja das Bekenntnis, das gerade damals entstanden sein und sich durchgesetzt haben muß. Dabei verdient wohl die Form der Erzählung bei Markus (12, 28-34), mit der die bei Matthäus (22, $34-40$ ) in der Hauptsache übereinstimmt, zunächst den Vorzug vor der etwas ungeschickten Form des Lukas (10, 25-28), wo ein Gesetzeslehrer zuerst seine Frage stellt, um sie sofort selbst auf das glänzendste $z u$ beantworten. Auffallen muß aber, daß keine der drei Darstellungen erkennen läßt, wie Jesus mit der Wahl von Dt $6,4.5$ nur seine Zustimmung zu einer ihm wie allen seinen Volksgenossen längst vertrauten Gewöhnung gibt. Vielmehr macht die Antwort des Schriftgelehrten bei Markus 12, 32.33 den Eindruck, als ob dem Schriftgelehrten etwas durchaus Neues entgegengebracht worden sei, das er sich überlegt. Darf man also auch auf die überlieferte Form von Rede und Gegenrede bei allen drei Evangelisten kein allzu großes Gewicht legen, so bleibt doch bestehen, daß die drei Erzähler bei dem Worte Jesu von einem Anschluß an eine bestehende jüdisch-religiöse Übung nichts $\mathrm{zu}$ wissen scheinen.

Aber wie wirkt diese Geschichte in der ersten Christenheit weiter?

Es fältt auf, daß Paulus zweimal (Rom 13,9; Gal 5, 14) das Gebot der Nächstenliebe als die Summe aller Gebote bezeichnet, ohne daneben das Gebot der Gottesliebe zu erwähnen. Nach der Erzählung der Evangelien hat Jesus dieses Gebot der Nächstenliebe dem Gebote der Gottesliebe Dt $6,4.5$ gleichgestellt. Aber hierin hat Lukas eine glaubwürdige Überlieferung über die Darstellung der andern hinaus. Nach ihm erklärt und veranschaulicht Jesus im Anschluß an den vorausgestellten Bescheid das Gebot der Nächstenliebe durch das Samaritergleichnis. Dazu vergleiche man bei Markus 10,19 die Aufzählung der Gebote an den Reichen, der. Jen Weg zum ewigen Leben wissen will. Jesus nennt die Gebote der zweiten Tafel und von den Geboten der ersten nur das Gebot, die Eltern zu ehren; die streng gottesdienstlichen Gebote läßt er weg. Zusammen mit dem Befund bei. Paulus dürfte das zeigen, daß Jesus die Hervorhebung von Lev 19, 18 mehr am Herzen lag und wichtiger war, als die Betonung von Dt 6,4.5. Und damit könnte auch der Evangelist Lukas zu seinem Recht kommen, wenn er sagt, daß der Schriftgelehrte selbst das höchste Gebot gekannt habe. In der dem Evangelisten vorliegenden Überlieferung bezog sich das 
wohl nur auf $\mathrm{Dt} 6,4.5$. Man darf die Vermutung aussprechen, daß Jesus davon wuBte, wie Dt $6,4.5$ als Glaubensbekenntnis von den Schriftgelehrten empfohlen worden ist. $\mathrm{Da}$ erklärt er sich mit dieser Entscheidung einverstanden, stellt aber dem Gebot der Gottesliebe das Gebot der Nächstenliebe als gleichivertig zur Seite.

Man gewinnt einen starken Eindruck von dem religiösen Leben im Judentum jener Tage, wenn man sich vorhält, daß in derselben Zeit das Christentum aus dem Schoß des Judentums hervorging und auch das jüdische Glaubensbekenntnis aufgestcllt und sein regelmäßiges Aufsagen durchgeführt wurde. Trotz dieser Gleichzeitigkeit braucht das jüdische Bekenntnis keinen Gegensatz zum Christentum zu betonen: zu der Zeit, als es aufkam, schrieb man jüdischerseits dem Christentum noch keine Lebenskraft $\mathrm{zu}$; vergleiche das Urteil der rö-

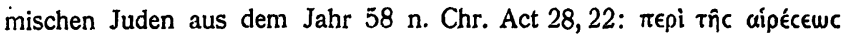

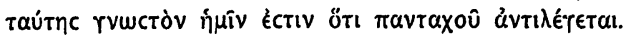

Für das Judentum gab es also seitdem eine angeblich im Gesetz begründete Pflicht, dieses Bekenntnis vor dem Einschlafen und wieder beim Erwachen, also abends und morgens, zu sprechen. „Beten“ nennt das der Jude allerdings nicht, sondern "aufsagen" (Nרָק). Aber das Judentum hat schon zur Zeit der Mischna das eigentliche Bekenntnis mit mancherlei Gebeten umrahmt; es sind abends andere als morgens, und auch sie sind genau festgestellt; so ist tatsächlich aus dem , Bekennen' schon frühe ein "Beten" geworden (Berachoth 1,4;2,2).

Das jüdische Bekenntnis in seiner vollen Form haben die Christen niemals übernehmen können; es schärft den Gebrauch der Gebetsriemen, der Türpfostensprüche, der Quasten am Kleide ein. Diese Dinge sind ja ab und $z \mathrm{u}$ von Christen nachgeahmt worden (vgl. Schürer II, 4. Aufl., $567,79)$; allgemeine Geltung haben sie in der Kirche nie erhalten. Auch konnte für Christen Lohn und Strafe der Frömmigkeit nicht mehr in Besitz oder Verlust Palästinas und der Fruchtbarkeit seines Bodens bestehen. Aber das Bekenntnis zur Einheit Gottes und zur Pflicht der Gottesliebe war den Christen so heilig wie den Juden; das Wort Jesu verlangte nur, daß neben dieses höchste Gebot das ihm gleichwertige der Nächstenliebe gestellt sei. Lernten also die Juden damals ihr $\mathrm{Be}-$ kenntnis morgens und abends sprechen, so lag es nahe genug, da $\beta$ auch die Christen das ihnen hüchste Gebot im Gesetz jeden Morgen und jeden Abend aufsagten. Auch sie mochten das nach Dt 6,7 als eine gesetzliche Pflicht betrachten. Tertullian, de orat. 25 zeigt deutlich, wie eifrig man in der Kirche bemüht war, nicht hinter den Juden zurückzustehen: aus dem "dreimal" bei Dan 6,10 wird ihm ein "nicht 
weniger als dreimal“ für die Christen („ne minus ter die saltem adoremus"). Auch Cyprian, de orat. domin. 34 verweist die Christen auf das alttestamentliche Vorbild.

Die Worte Tertullians "exceptis utique legitimis orationibus, quae sine ulla admonitione debentur ingressu locis et noctis" beziehen sich also nicht auf das Beten des Vaterunsers, sondern auf das Sprechen eines dem jüdischen ähnlichen, aus dem Gesetz entnommenen Bekenntnisses, vielleicht nur der Worte Dt 6,4.5; Lev 19,-18. Möglich ist, daß auch die Christen diese Worte mit eigentlichen Gebeten umrahmt haben.

5. Die "gesetzlichen Gebete beim Eintritt ron Tag und Nacht" rechnet Tertullian nicht ein, wenn er mindestens drei Tagesgebete fordert und dafür die dritte, sechste und neunte Stunde empfiehlt (de orat. 25; de jejun. 10). Als Grundlage dieses Gebetes betrachtet er das Vaterunser, an das man besondere Einzelwünsche nach der jedesmaligen Lage anschließen mag (de orat. 10). Hierin trifft er mit der Didache zusammen, die ein täglich dreimaliges Beten des Vaterunsers vorschreibt $(8,3)$. Auch Clemens von Alexandrien kennt nach Strom. VII, 7, 40, 3 Leute, welche ein Gebet in der dritten, sechsten und neunten Stunde fordern. In der spätern Kirche (Cyprian, de orat. dom. 34; Constit. apost. VIII, 34) sind die dritte, sechste und neunte Stunde als Gebetsstunden festgehalten worden. Dagegen nennt Origenes, de orat.12 Morgen, Mittag und Abend als Gebetszeiten.

Für die dritte, sechste und neunte Stunde sprach nach Tertullian die römische Tageseinteilung, wie sie schon von Varro, de ling. Lat. VI, 89 bezeugt ist. Dazu kamen die Angaben der Apostelgeschichte vom Pfingstwunder um die dritte Stunde, von der Offenbarung an Petrus bei seinem Gebet um die sechste und von der Lahmenheilung durch die zum Tempel gehenden Apostel und vom Gebet des Hauptmanns Cornelius un die neunte Stunde (Act 2,$15 ; 10,9 ; 3,1 ; 10,3.30$ ). Das jüdische Vorbild des Daniel ruft 'Tertullian nur für die Zahl der täglichen Gebete an (Dan. 6, 10).

Die römische Tageseinteilung mit.öffentlicher Verkündigung der dritten, sechsten und neunten Stunde erleichterte die Einführung und Einbürgerung dieser Gebetsstunden. Aber auch Anlehnung an die jüdische Sitte hatte statt. Das dürfte daraus hervorgehen, daß Act 3,1 die

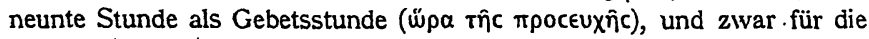
jüdische Welt, geradezu bezeichnet wird. Ebenso spricht der Hauptmann Cornelius deutlich von einer festen Sitte, ivenn er Act 10, 30 er-

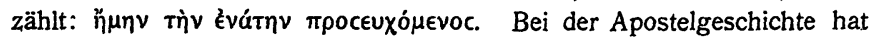
man ja gewiß Grund, behutsam zu sein. Mañ könnte sich vorstellen, 
daß hier die christliche Sitte in das Judentum zurückgetragen ist, zumal da der freie Verkehr der nächsten Jünger Jesu im Tempel so bald nach der Verurteilung Jesu im Hohenrat - recht unwahrscheinlich ist. Aber die neunte Stunde als jüdische Gebetsstunde steht dennoch fest.

Nach Jos., antt. 14, 65 wurden dic beiden täglichen Gemeindeopfer, die den gesamten Tagesdienst im Tempel umschlossen, in der Morgen-

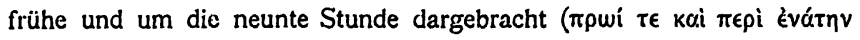
w̋pav). Das Morgenopfer beginnt mit einem Rauchopfer im Tempelhause, das Nachmittagsopfer schließt damit (Philo, de victimis 3, de sacrificantibus 4). Lk 1,9.10 wird es zur feststehenden Sitte gerechnet, daß während des täglichen Rauchopfers im Tempelhause die im Tempel-

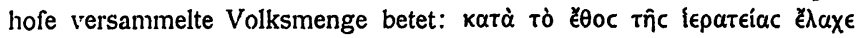

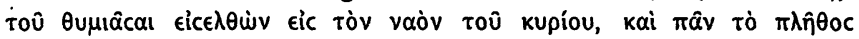

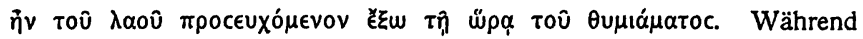
des abendlichen Rauchopfers in Jerusalem beten auch die Juden außerhalb Jerusalems nach Judith 9,1 . Und von dieser Sitte her heißt das tägliche Nachmittagsgebet in der Mischna und demgemäß noch heute

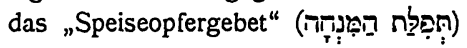

Das abendliche Gemeindeopfer war nämlich ursprünglich nur ein Speiseopfer (Kuchenopfer) gewesen, während morgens schon in altisraelitischer Zeit ein Brandopfer dargebracht wurde; so unterschied man damals das Morgenbrandopfer vom Abendspeiseopfer (II Kön 16, 15). Demgemäß sprach schon das alte Israel von der Zeit, da man das Speiseopfer bringt (I Kön 18, 29). Dieser Sprachgebrauch wurzelte so fest, daß er bestehen blieb, als das Gesetz für Morgen und Abend dieselben Rauchopfer, Brandopfer, Speiseopfer und Trankopfer forderte (Ex 29, 38-42; Num 28, 3-8), ja als man diese neue Sitte schon in die alte Zeit zurückverlegte (I Chron 16,40; II Chron 13,11). Noch Esra und Daniel beten „zur Zeit des Speiseopfers“ Ex 9, 4; Dan 9, 21. Und diesen Sprachgebrauch halten die Juden noch heute fest. Die Zeit des Nachmittagsopfers blieb im Gedächtnis, auch als das Opfer nicht mehr gebracht wurde. Nach der Zeit des hadrianischen Krieges wirkt Rabbi Juda ben Elai (s. Strack, Einleitung in den Talmud, 4. Aufl., S. 93): er forderte, daß man um die Mitte des Speiseopfers das Speiseopfergebet gesprochen habe (Berachoth 4, 1). Damit weicht er von der alten Sitte ab, die das Gebet zur Zeit des Rauchopfers verlangt, das den Abschluß des Nachmittagsopfers bildet. Aber merkwürdig bleibt, daß hier sogar nach Teilen des Speiseopfers gerechnet wird. - Das alttestamentliche Psalmbuch enthält ein Speiseopfergebet Ps 141, wo es v. 2 heißt: 
Stell' mein Gebet als Rauchopfer vor dich,

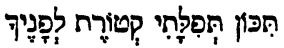

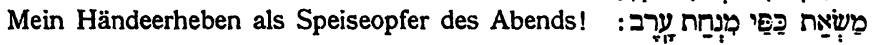

Wie sehr Rauchopfer und Gebet für jüdisch gebildetes Denken zusammengehören, zeigt auch die Offenbarung Johannis, wo das Rauchwerk in den goldenen Schalen vor Gottes Thron die Gebete der Gläubi-

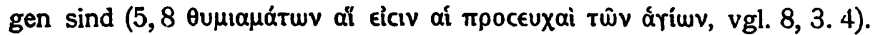

Die jüdische Sitte der Gebetsstunden knüpft also an die Opferordnung des Jerusalemer Tempels an. Beim täglichen Gemeindeopfer ist die Gemeinde mit ihrem Gebet zugegen. Auch wer nicht in Jerusalem weilt oder nicht in den Tempel kommen kann, betet doch mit der übrigen Gemeinde zu dieser Zeit. Daher erklärt sich auch die Sitte, die schon Dan 6,10 bezeugt ist, beim Gebet nach Jerusalem zu blicken; Daniel hat nach dieser Stelle in seinem Hause sogar eigenes Fenster in der Richtung nach Jerusalem. Der Betende gliedert sich gleichsam der dort im Tempelhof weilenden Gemeinde an und blickt mit ihr nach dem Tempel empor, wo der Priester im Rauchopfer die Gebete der Gemeinde vor Gott bringt. Die Mischna gibt über die Gebetsrichtung ganz genaue Bestimmungen (Berachoth 4,5.6).

Damit ist für die jüdische Sitte ein Frühgebet beim Morgenopfer und ein Nachmittagsgebet festgestellt. Gegenüber den alttestamentlichen Aussagen und Bestimmungen muß nun aber die durch Josephus und die Apostelgeschichte festgestellte Zeit des Speiseopfers auffallen. Denn nach allen alttestamentlichen Angaben ist die Zeit des zweiten täglichen Gemeindeopfers der Abend; nach Josephus und der Apostelgeschichte ist es die neunte Stunde, also der Nachmittag; und auch die Mischna unterscheidet die Zeit des Speiseopfers und den Abend

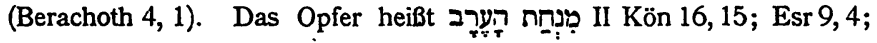
Dan 9, 21; Ps 141, 2; vgl. II Chron 2, 3; 31,3; Esr 3, 3. Im Gesetz ist als

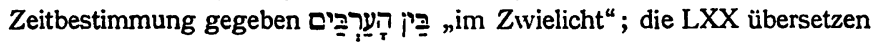

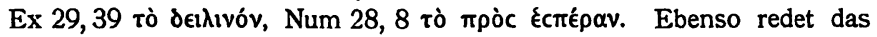

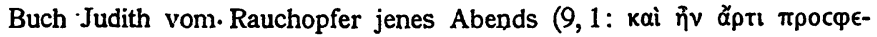

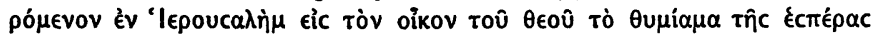

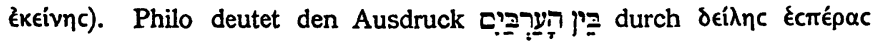
und meint, daß das Opfer bei-Sonnenuntergang sein Ende finde (íniou ठuopévou): de victimis 3. Durch das Frühopfer, sagt er, dankt man für die Segnungen des Tags und durch das Abendopfer für die der

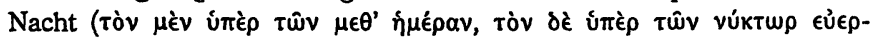
reciŵv). Nach Ex 30, 7.8 soll das Rauchopfer im Tempel mit der Besorgung des Leuchters morgens und abends verbunden sein; abends 
fand es also ursprünglich statt bei eintretender Dunkelheit: (im Zwiclicht). Die von Josephus, der Apostelgeschichte und der Mischna bezcugte Sittc, das Abendopfer schon um die neunte Stunde darzubringen, ist eine Neucrung, die wohl erst im herodianischen Tempel durchgeführt wurde, als die großen Anforderungen und vielleicht auch die Bequemlichkeit der Priesterschaft eine Einschränkung des täglichen Dienstes wünschenswert machten. Die Anforderungen waren gewachsen bei der Leichtigkeit des Verkehrs innerhalb des großen Reichs, das sich unter den ersten Kaisern eines dauernden Friedens erfreute; bei dem Ansehen, das die Familie des Herodes dem Judentum innerhalb des Reichs zu verschaffen wußte; auch bei der Pracht des neuen Tempels, der die Fremden anlockte; nicht zum mindesten endlich durch die rührige Propaganda, die wenigstens im ganzen Osten des Reichs den jüdischen Gemeinden immer neue Mitglieder zuführte (vgl. Mit 23,15). Das alles brachte dem Tempel neue Einkünfte, aber vermehrte auch die Arbeit der Priester; so setzten sie es durch, daß das Abendopfer schon um 3 Uhr nachmittags dargebracht und dann der Tempel geschlossen wurde. Mit dem Abschluß der Tempeltore hatte der Dienst der Priester für diesen Tag in der Hauptsache sein Ende gefunden (Joseph., contr. Apion. II, 105. 119; bell. Jud. VI, 293).

Daraus geht hervor, daß es nicht der alten Sitte entsprechen kann, wenn die Mischna und ihr folgend das spätere Judentum nach dem "Speiseopfergebet" noch ein späteres „Abendgebet" fordern (Berachoth4,1). Wenn man beim Speiseopfer nach Philo, de victim. 3 für die Wohltaten der Nacht dankte, wenn das Speiseopfer ausdrücklich immer als Abendopfer bezeichnet wurde, so hat neben dem Speiseopfergebet ein besonderes Abendgebet keinen Raum. Dazu kommt, daß man auch beim dritten Gebet w'ie beim Morgen- und Speiseopfergebet seinen Blick schon zu Daniels Zeit (Dan 6,10) nach Jerusalem richtete. Aber nach dem Speiseopfer gab es in dem verschlossenen Tempel keine feiernde Gemeinde, in die man sich mit seinen Gedanken hätte einrechnen können. Die Analogie mit Frühgebet und Speiseopfergebet fordert auch für dieses dritte Tagesgebet einen bestimmten Anlaß in der Ordnung des täglichen Tempeldienstes. Auch dieses dritte Gebet muß ursprünglich zu einer Zeit gesprochen worden sein, in welcher der Tempel regelmäßig von einer betenden Gemeinde besucht war.

Ehe diese ursprüngliche dritte Gebetsstunde festgestellt werden kann, muß noch ein Punkt aufgewiesen werden, an welchem die Bestimmung der Mischna für die Zeit vor Zerstörung des Tempels sicher nicht zutrifft. Nach Berachoth 4,1 darf das Frühgebet bis. zur Mittags- 


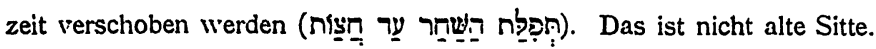
Bei dem engen Zusammenhang zwischen diesem Gebet und dem in der Morgenfrühe dargebrachten Rauchopfer im Tempel war ein so langes Hinausziehen des Morgengebetes von der strengen Sitte gewiß nicht gestattet. Es konnte ein solches Hinausziehen erst gestattet werden, als mit der Aufhebung des Opferdienstes das Gebet seinen Halt an der Ordnung des Tempels verloren hatte.

Gegen die Mischna und das ihr folgende Herkommen ist also daran festzuhalten, daß, solange der Tempeldienst dauerte, w'eder das Frühgebet bis zum Mittag verschoben, noch ein Abendgebet nach dem Speiseopfergebet gesprochen wurde; das Aufsagen des Bekenntnisses ist ja kein Gebet. Gegen diese Auffassung könnte geltend gemacht werden, daß Judith nachts betet $(11,17 ; 12,5-9 ; 13,10)$. An das Aufsagen des Bekenntnisses darf man hier auch deshalb nicht denken, da die Frau von dieser Pflicht gewiB immer befreit war (Berachoth 3,3); auch ist ausdrücklich rom Gebet die Rede. Aber Judith lebt im heidnischen

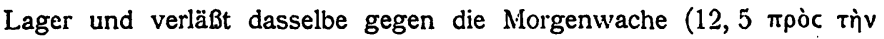

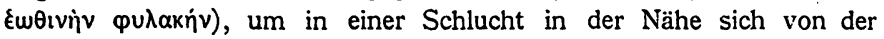
Befleckung durch das Heidentum reinzubaden (12, 6-9): dafür ist in ihrer Lage die Nacht die geeignete Zeit. Aus diesen Stellen wird man also schwerlich auf eine nächtliche Gebetszeit der Juden schließen dürfen.

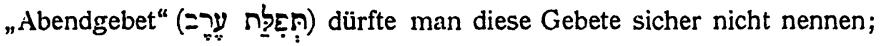
eher sind sie ein frühes Morgengebet. Nach Philo (de sacrif. 4) sollte das Rauchopfer morgens $\pi \epsilon \hat{\mathrm{i}} \beta \alpha \theta \dot{\mathrm{u} v}$ ő $\rho \theta \rho o v$ stattfinden.

Nun heißt es Ps 55,17.18: „Ich will rufen zu Gott und Jahve hilft mir; abends, morgens und mittags will ich reden und seufzen, und er hört meine Stimme." Ebenso heißt es in der von Dalman als babylonisch bezeichneten Fassung des jüdischen Tagesgebetes, die noch heute im wesentlichen von den Juden überall gebetet wird (im Unterschied von der in Ägypten wiedergefundenen "palästinischen " Fassung, welche diese Worte nicht hat; s. Dalman, Worte Jesu, S. 300), im sogenaninten Dank (St. 18): „Wir danken dir und erzählen deinen Ruhm - - - für deine Güte zu jeglicher Zeit, am Abend, am Morgen, am Mittag." Auch wenn man das so versteht, daß Gott immerdar seine Güte erweist, also in - den drei besonders genannten Tagesabschnitten, so ist doch klar, daß man dann auch in diesen drei Tagesabschnitten Gott für seine Güte zu danken hat. Der Wortlaut des Achtzehngebetes steht hier also im Gegensatz zu der von der Mischna geheiligten Sitte, die von einem Mittagsgebet nichts weiß. Ferner nennt noch Epiphanius, haer. 29, 9 (opp. ed. Dindorf II, p. 89) Morgen, 
Mittag und Abend geradezu als die jüdischen Gebetszeiten, wie sie in der Gegenwart des Epiphanius gehalten würden: àvictú $\mu \in v o ı ~ " z w \theta \in v$

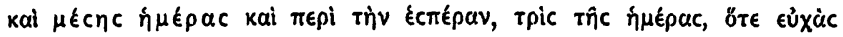

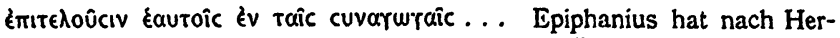
kunft und Wohnort die jüdische Sitte Palästinas, Ägyptens und Cyperns vor Augen. In diesen Gegenden hat also die Sitte eines täglichen Mittagsgebetes neben Frühgebet und Abendgebet bis um $400 \mathrm{n}$. Chr. fortbestanden. Auch die Apostelgeschichte erwähnt ja dieses Mittagsgebet um die sechste Stunde $(10,9)$. P. Drews hat also recht, wenn er zu Didache 8, 3 bemerkt, daß die Juden das Schmone Esre morgens, mittags und abends gebetet haben.

Während sich für ein weiteres Abendgebet nach dem abendlichen Rauchopfer in der Sitte des Tempels keinerlei Anlaß findet, hatte ein Gebet um die Mittagszeit in der Tempelsitte seinen guten Grund. Nach einem Anlaß in der Tempelsitte muß man aber auch für dieses dritte Gebet suchen, da es wie die beiden andern mit Richtung des Betenden nach dem Tempel schon zur Zeit des Danielbuches gesprochen wurde (Dan 6, 10). Die Mittagsstunde war für den Gottesdienst bedeutsam. Nach Josephus, contr. Apion. II, 105 betreten auch die Priester nur zu bestimmter Tageszeit den ihnen vorbehaltenen Raum des Tempels beim Altar: nämlich frühmorgens und um die Mittagszeit. Es scheint vorausgesetzt zu sein, daß nach der Darbringung der Opfer am Vormittag eine Unterbrechung des Opferdienstes eintritt; erst am Mittag wird die Opferarbeit wieder aufgenommen und dauert dann fort, bis der Tempel geschlossen wird: tanta vero est circa omnia providentia pietatis, ut secundum quasdam horas sacerdotes ingredi constitutum sit: mane etenim aperto templo oportebat facientes traditas hostias introire et meridie rursus, dum clauderetur templum. Somit war die Mittagsstunde als Zeitpunkt des Wiedererscheinens der Priester und der Wiederaufnahme der Opfer ein bedeutsamer Augenblick für den Tempel. Da füllte sich auch der Tempelhof wieder, der vorher eine Zeitlang verlassen war. Auf solche Tempelsitte weist auch der in demselben Kapitel des Josephus (c. Apion. II, 108 mediante die) bezeugte Wechsel der diensttuenden Priesterabteilungen am Mittag jedes Sabbates hin (vgl. Joseph. antt. VII, 365). Wie also das Morgengebet beim täglichen Morgenopfer den Tagesdienst. im Tempel einleitete, so bezeichnete auch das Mittagsgebet den Wiederbeginn der Opfer, die mit dem Speiseopfergebet ihren feierlichen Abschluß fanden.

Aus Epiphanius, haer. 29, 9 geht also hervor, daß die Juden in der Welt des Epiphanius noch ganz nach der alten Tempelsitte mor- 
gens, mittags und abends ihr Gebet sprachen. In der Apostelgeschichte finden wir das Mittagsgebet um die sechste Stunde $(10,9)$ festgehalten neben dem nach der letzten Tempelsitte (Jos., antt. 14, 65) auf die neunte Stunde zurückgeschobenen Speiseopfergebet (Act 3,1;10,3.4). Auch Tertullian weiß de jejun. 10, daß das Gebet des Cornelius um die neunte Stunde sein drittes und damit letztes Tagesgebet gewesen ist. Weniger sicher ist, ob die Apostelgeschichte die dritte Stunde als Zeit des Morgengebetes betrachtet. Es mag naheliegen, das Wort 2,15 so zu verstehen, besonders da die Zungenrede tatsächlich nach Paulus immer Gebetsrede gewesen ist (1 Kor 14, 2.13-16. 28). Aber die Apostelgeschichte redet bei ihrer Darstellung des Pfingstereignisses durchaus nicht von einer Gemeinde, die zum Gebet zusammengekommen ist; vielmehr ist 2,6 ausdrücklich gesagt, daß das Wunder die Menge zusammengeführt habe. Unmöglich wäre es nicht, daß im herodianischen Tempel das Morgenopfer erst un die dritte Stunde gehalten vurde, so daß man auch da erst zum Beten zusammenkam. Denn die Mischna erlaubt die Verschiebung des Morgengebetes bis um die Mittagsstunde; dabei erwähnt sie noch die strengere Meinung des Rabbi Juda, der es nur bis zur vierten Stunde (also $10 \mathrm{Uhr}$ ) verschoben wrissen wollte. Die dritte Stunde hätte Rabbi Juda - ein Schriftgelehrter zwischen 130 und $160 \mathrm{n}$. Chr. - also sicher für die ordnungsmäßige Zeit des Frühgebets gehalten (Berachoth 4,1). Man kann noch von anderer Seite zu ähnlichem Schlusse kommen. Dem Morgengebet mußte immer das Morgenbekenntnis vorausgehen. Da erlaubt Rabbi Josua (um 100 n. Chr.), das Morgenbekenntnis noch um die dritte Stunde aufzusagen, weil Königskinder erst um diese Zeit aufstehen (Berachoth 1,2). Also schien auch ihm für das Morgengebet die dritte Stunde nicht zu spät zu sein; und doch mochte man sich zu seiner Zeit der Tempelsitte noch wohl erinnern (vgl. über ihn Strack, Einl. in den Talmud, 4. Aufl., S. 87). War die dritte Stunde die Stunde des Morgengebetes, so war der Opferdienst im Tempel schließlich auf die Zeit etwa von acht Uhr morgens bis vier Uhr abends mit einer Unterbrechung vor Mittag beschränkt. Das ist bei der Verweltlichung des Priestertums in jener Zeit nicht undenkbar. Aber die dritte Stunde ist nirgends sicher als die Zeit des Morgenopfers oder Morgengebetes bezeichnet.

Nun nennt Origenes, de orat. 12 als Gebetszeiten der Christen Morgen, Mittag und Abend, genau wie Epiphanius, haer. 29, 9 (neben Ps 55, 18 und Achtzehngebet [bab.] 18) dieselben Gebetszeiten für die Juden bezeugt. Hier liegt doch handgreiflich auf seiten der Kirche Anlehnung

Zeitschr. f. d. neutest. Wiss. XII. Jahrg. 1911. 
an die jüdische Sitte vor. Ebenso ist aber auch Anlehnung an eine etwas andere Gestalt dieser jüdischen Sitte vorhanden, wenn Tertullian, de orat. 25 und de jejun. 10, Clemens von Alexandrien, Strom. VII, 7, 40, 3, Cyprian, de orat. dom. 34 die dritte, sechste und neunte Stunde als regelmäßig festzuhaltende Gebetszeiten bezeichnen. Für die sechste und neunte Stunde konnte das sicher, für die dritte mit einiger Wahrscheinlichkeit nachgewiesen werden. Immerhin könnte bei der dritten Stunde auch die bürgerliche Tageseinteilung (Tertullian, de or. 25, de jejun. 10; Varro, de Lingua Latina VI, 89) und der Wunsch, einen zeitlichen Abstand vom Gebet beim Erwachen zu erreichen, maßgebend gewesen sein. Denn wie Tertullian, de orat. 25, so will auch Cyprian außer den drei feststehenden ordnungsmäßigen Gebeten noch ein Frühgebet und ein Abendgebet; ja er fordert noch dazu in unbestimmter Weise nächtliche Gebete (de orat. dom. 35). Die apostolischen Konstitutionen (VIII, 34) ordnen das so, daß sie neben den drei Tagesgebeten zur dritten, sechsten und neunten Stunde ein Morgen- und Abendgebet und ein Gebet beim Hahnenșchrei fordern. Das Gebet am Frühmorgen mußte also vom feierlichen ersten Tagesgebet getrennt werden. Das mag dazu beigetragen haben, daß letzteres auf die dritte Stunde gelegt wurde.

6. Die Anlehnung der christlichen Sitte an die jüdische hinsichtlich der Gebetsstunden wäre leichter erkennbar geblieben, wenn nicht das Judentum nach der Zerstörung des Tempels seine Gebetssitte geändert und wenn diese Änderung sich nicht mit der Herrschaft der Mischna allmählich in der ganzen jüdischen Welt durchgesetzt hätte. Schürer hat hier wie in allen ähnlichen Fällen die Angabe der Mischna auch für das Zeitalter Jesu für maßgebend gehalten. Man liest in seiner Geschichte des jüdischen Volkes im Zeitalter Jesu Christi, 4. Aufl., II, 350, 40: „Ganz verkehrt ist die auf Mißverständnis von Act 2,$15 ; 3,1 ; 10,3.9 .30$ beruhende Meinung, daß je um die dritte, sechste und neunte Stunde (also nach unserer Zählung um neun, zwölf und drei Uhr) eine ständige Gebetszeit gewesen sei (so z. B. Schoettgen, Horae hebr. I, 418; Winer RWB I, 398; De Wette zu Act 2, 15; Meyer zu Act 3,1). Die wirklichen drei Gebetszeiten waren vielmehr: 1. frühmorgens zur Zeit des Morgenopfers, 2. nachmittags um die neunte Stunde (drei Uhr) zur Zeit des Abendopfers, 3. abends zur Zeit des Sonnenuntergangs. S. Berachoth I, If.; IV, 1."

Von den beiden Mischnastellen, die Schürer hier anführt, redet zwar die erste überhaupt nicht vom Gebet (苂), sondern vom Bekenntnis (שֵֶׁ) Die zweite lautet allerdings: „Das Morgengebet bis 
Mittag; Rabbi Juda sagt: bis zur vierten Stunde. Das Gebet des Spciseopfers bis zum Abend; Rabbi Juda sagt: bis zur Mitte des Speiseopfers. Das Abendgebet hat keine Regel." Auch das stimmt ja mit Schürers Worten nicht ganz überein. Von "frühmorgens" und dem "Morgenopfer" und von der "Zeit des Sonnenuntergangs" steht hier nichts. Aber das Mittagsgebet ist ausgeschaltet, und als drittes Gebet ist ein Abendgebet eingeführt, das die ganze Nacht hindurch ge-

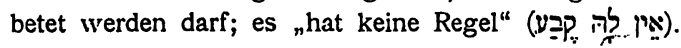

Es ist gezeigt worden, daß und warum diese Ordnung nicht ursprünglich ist. Das Speiseopfergebet war ursprünglich das Abendgebet, mit dem der tägliche Gottesdienst im Tempel abschloß; ein Mittagsgebet ist mehrfach bezeugt und war in der Sitte des Tempels begründet. Also bleibt noch übrig, die Gründe für die Änderung der alten Sitte aufzusuchen. Maßgebend dürfte hier die Verlegung des Speiseopfers vom Abend auf den Nachmittag gewesen sein. Ein Gebet um drei Uhr nachmittags wurde eher als ein verspätetes Mittagsgebet, denn als ein frühes Abendgebet aufgefaßt. In Jerusalem hielt man freilich, solange der Tempeldienst bestand, an der Sitte des Tempels fest und betete um drei Uhr das letzte Tagesgebet, nachdem man um zwölf Uhr das Mittagsgebet gesprochen hatte. Und nach Jerusalem richtete sich Judäa. Diese Ordnung finden wir in der Apostelgeschichte (Act 3, 1; 10, 3.4. 30; 10, 9; Tertullian, de jejun. 10); die Apostelgeschichte gibt damit die Verhältnisse der geschilderten Zeit in durchaus zuverlässiger Weise wieder. In der Diaspora und nach dem Fall des Tempels hielt man entweder an der früheren Sitte fest, morgens, mittags und abends zu beten (Ps 55, 18 - Achtzehngebet [bab.] 18; Epiphanius, haer. 29,9); oder man änderte die Gebetszeiten so, daß man an Stelle des Mittagsgebetes das Speiseopfergebet um drei Uhr setzte, das Abendgebet aber nach wie vor festhielt. Das war um so leichter möglich, als das Mittagsgebet wohl in einer feststehenden Tempelsitte, nicht aber in dem durch das Gesetz geheiligten Brauch des täglichen Gemeindeopfers seinen Grund hatte. Șo bestanden beide Gebetsordnungen bis über die Zeit des Epiphanius hinaus im Judentum nebeneinander. Durch die Autorität der Mischna wurde die ältere schließlich verdrängt. Dagegen behielt die Kirche das.Gebet um die sechste neben den Gebeten um die dritte und neunte Stunde bei. 